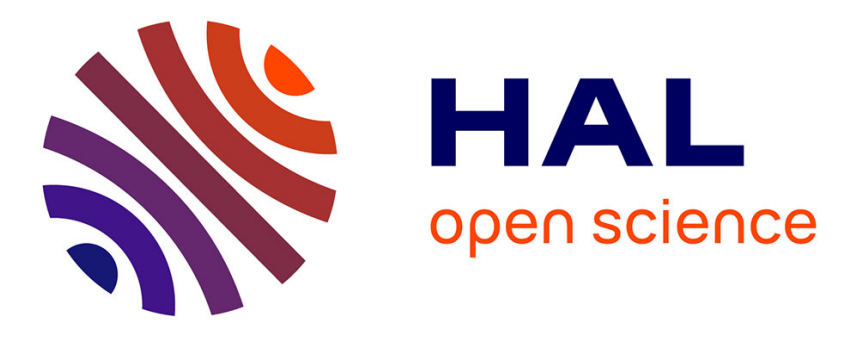

\title{
Synthesis of fractional Laguerre basis for system approximation
}

Mohamed Aoun, Rachid R. Malti, François Levron, Alain Oustaloup

\section{To cite this version:}

Mohamed Aoun, Rachid R. Malti, François Levron, Alain Oustaloup. Synthesis of fractional Laguerre basis for system approximation. Automatica, 2007, 43 (9), pp.1640-1648. 10.1016/j.automatica.2007.02.013 . hal-00180684

\section{HAL Id: hal-00180684 https://hal.science/hal-00180684}

Submitted on 20 Oct 2007

HAL is a multi-disciplinary open access archive for the deposit and dissemination of scientific research documents, whether they are published or not. The documents may come from teaching and research institutions in France or abroad, or from public or private research centers.
L'archive ouverte pluridisciplinaire HAL, est destinée au dépôt et à la diffusion de documents scientifiques de niveau recherche, publiés ou non, émanant des établissements d'enseignement et de recherche français ou étrangers, des laboratoires publics ou privés. 


\title{
Synthesis of fractional Laguerre basis for system approximation
}

\author{
M. AOUN ${ }^{a, b}$ R. MALTI ${ }^{a}$ F. LEVRON ${ }^{c}$ A. OUSTALOUP ${ }^{a}$ \\ a IMS, UMR 5218 CNRS, Département LAPS - Université Bordeaux 1 \\ 351 cours de la Libération - F 33405 Talence cedex - France \\ ${ }^{\mathrm{b}}$ MACS - École Nationale d'Ingénieurs de Gabès \\ Rue Omar Ibn El Khattab - T 6029 Gabès - Tunisia \\ ${ }^{\mathrm{c}} I M B, U M R 5251$ CNRS - Université Bordeaux 1 \\ 351 cours de la Libération - F 33405 Talence cedex - France
}

\begin{abstract}
Fractional differentiation systems are characterized by the presence of non-exponential aperiodic multimodes. Although rational orthogonal bases can be used to model any $L_{2}[0, \infty[$ system, they fail to quickly capture the aperiodic multimode behavior with a limited number of terms. Hence, fractional orthogonal bases are expected to better approximate fractional models with fewer parameters. Intuitive reasoning could lead to simply extending the differentiation order of existing bases from integer to any positive real number. However, classical Laguerre, and by extension Kautz and generalized orthogonal basis functions, are divergent as soon as their differentiation order is non-integer. In this paper, the first fractional orthogonal basis is synthesized, extrapolating the definition of Laguerre functions to any fractional order derivative. Completeness of the new basis is demonstrated. Hence, a new class of fixed denominator models is provided for fractional system approximation and identification.
\end{abstract}

Key words: Orthonormal basis, fractional differentiation, Laguerre function, system approximation, identification.

\section{Nomenclature}

$(.)^{T} \quad$ transpose of a matrix.

$\lfloor\cdot\rfloor \quad$ floor operator.

$\delta_{n m} \quad$ Dirac delta function equals 1 if $n=m$, and 0 otherwise.

$\mathbb{N} \quad$ set of non-negative integers.

$\mathbb{R} \quad$ field of real numbers.

$\mathbb{R}^{+} \quad$ set of non negative real numbers.

$\mathbb{R}^{+*} \quad$ set of strictly positive real numbers.

$\mathbb{C} \quad$ field of complex numbers.

Email addresses: mohamed.aoun@gmail.com ( M. AOUN), rachid.malti@laps.ims-bordeaux.fr ( R. MALTI), Francois.Levron@math.u-bordeaux1.fr ( F. LEVRON), alain.oustaloup@ims-bordeaux.fr ( A. OUSTALOUP).

\begin{tabular}{|c|c|}
\hline $\mathbb{C}^{+}$ & open right half-plane $\{s \in \mathbb{C}: \mathscr{R} e(s)>0\}$. \\
\hline$\overline{\mathbb{C}^{+}}$ & closed right half-plane $\{s \in \mathbb{C}: \mathscr{R} e(s) \geq 0\}$. \\
\hline$L_{2}[0, \infty[$ & $\begin{array}{l}\text { Lebesgue space of squared integrable func- } \\
\text { tions in }[0, \infty[\text {. }\end{array}$ \\
\hline$H_{2}\left(\mathbb{C}^{+}\right)$ & $\begin{array}{l}\text { Hardy space of functions } F \text {, analytical on } \\
\mathbb{C}^{+} \text {and continuous on } \overline{\mathbb{C}^{+}} \text {such that }\|F\|_{2}= \\
\frac{1}{2 \pi} \int_{-\infty}^{\infty}|f(x+j y)|^{2} d y<\infty .\end{array}$ \\
\hline$\Gamma(m)$ & $\begin{array}{l}\text { Euler's Gamma function, defined as } \Gamma(m)= \\
\int_{0}^{\infty} e^{-x} x^{m-1} d x \forall m \in \mathbb{R} \backslash\{0\} .\end{array}$ \\
\hline $\mathscr{C}$ & Cholesky decomposition \\
\hline
\end{tabular}

\section{INTRODUCTION}

Over the last fifteen years, orthogonal functions have been widely used in identification and control of linear systems; see for instance $[13,15,21,22]$ and their own references. The most popular orthogonal functions used in control engineering are Laguerre functions with a single 
pole, Kautz functions with two complex conjugate poles, and the Generalized Orthogonal Basis (GOB) functions. The latter extend the first two to any number of real or complex conjugate poles. All these bases span Lebesgue space of squared integrable functions, provided the completeness condition is satisfied [2]. They can hence approximate any linear system, including those with fractional differentiation, provided its impulse response belongs to $L_{2}[0, \infty[$. However, fractional systems, such as thermal [5] or electrochemical [7] systems (see the special issue of Signal Processing [17] for other fields of application), are characterized by the presence of two kinds of modes: exponential modes, as in rational systems, and aperiodic multimodes [18] which decay polynomially.

Using rational orthogonal bases to approximate a fractional $L_{2}[0, \infty$ [ system leads to a high truncation order to capture the non-exponential mode's behavior.

An intuitive approach to extend Laguerre functions to fractional differentiation orders led El-Sayed [8] to simply allow their differentiation orders to be positive real numbers without any precaution. However, Abbot [1], commenting on El-Sayed's work, has since proved that classical Laguerre functions are divergent whenever their differentiation order is non-integer.

In this paper, a fractional orthonormal basis is synthesized by applying the Gram-Schmidt orthogonalization procedure. The objective is twofold: to extrapolate Laguerre functions to any fractional derivative, and to obtain convergent functions forming a complete orthonormal basis in $H_{2}\left(\mathbb{C}^{+}\right)$. Hence, a new class of fixed denominator models is provided for fractional differentiation systems. The new orthonormal basis is governed by two tuning parameters: an eigenvalue and a commensurable order $\gamma \in] 0,2[$, (all differentiation orders are multiples of $\gamma$ ). Laguerre functions correspond to the special case $\gamma=1$. This is the first working fractional orthonormal basis ever synthesized.

\section{MATHEMATICAL BACKGROUND}

\subsection{Definition of fractional differentiation}

Differentiation to not only integer but also non-integer orders was defined in the $19^{t h}$ century by Riemann and Liouville. This is usually termed fractional differentiation. The $\gamma^{\text {th }}$ fractional derivative of a continuous real function $f(t)$ is defined as [16]:

$$
\begin{aligned}
& \mathbf{D}^{\gamma} f(t)= \\
& \frac{1}{\Gamma(\lfloor\gamma\rfloor-\gamma+1)}\left(\frac{d}{d t}\right)^{\lfloor\gamma\rfloor+1}\left(\int_{0}^{t} \frac{f(\tau)}{(t-\tau)^{\gamma-\lfloor\gamma\rfloor}} d \tau\right)
\end{aligned}
$$

The Laplace transform of $\mathbf{D}^{\gamma} f(t)$, when $f$ is relaxed at $t=0(f(t)$ and all its derivatives equal 0 for all $t<0)$, is given by [16]:

$$
\mathscr{L}\left(\mathbf{D}^{\gamma} f(t)\right)=s^{\gamma} F(s)
$$

This result is coherent with the classical case when $\gamma$ is an integer. Consequently, it is easy to define a symbolic representation of a fractional dynamic system using the transfer function:

$$
F(s)=\frac{\sum_{i=0}^{m_{A}} a_{i} s^{\alpha_{i}}}{1+\sum_{j=1}^{m_{B}} b_{j} s^{\beta_{j}}}
$$

where $a_{i}$ and $b_{j}$ belong to $\mathbb{R}$ and $\alpha_{i}$ and $\beta_{j}$ belong to $\mathbb{R}^{+}$, for $i=0,1, \ldots, m_{A}$, and for $j=1,2, \ldots, m_{B}$. Both $\alpha_{i}$ and $\beta_{j}$ form strictly increasing sequences.

$F(s)$ is called a commensurable transfer function at order $\gamma \in \mathbb{R}^{+*}$, iff $\left(\alpha_{i}, \beta_{j}\right)$ are commensurable 1 with $\gamma$. This $\gamma$ is called a commensurable order and the biggest number is always chosen.

Time-domain simulation of fractional transfer functions (3) is explained in $[3,4,19]$.

\subsection{Aperiodic multimodes as compared to exponential modes}

Taking the inverse Laplace transform of the following simple fractional transfer function $\frac{1}{s^{\gamma}-\lambda}$ gives [18]:

$$
\sum_{k=1}^{K} \frac{s_{k}}{\gamma \lambda} \mathrm{e}^{t s_{k}}+\frac{\sin (\gamma \pi)}{\pi} \int_{0}^{\infty} \frac{x^{\gamma} \mathrm{e}^{-t x}}{x^{2 \gamma}-2 \lambda x^{\gamma} \cos (\gamma \pi)+\lambda^{2}} d x
$$

where $s_{k}, k=1, \ldots, K$, are the $s$-roots of $s^{\gamma}-\lambda=0$. The number of $s$-roots, $K$, depends on $\gamma$ as shown in [18]. Expression (4) shows the presence of exponential modes in the left part, as in rational transfer functions. Also aperiodic multimodes, which decay polynomially, are present in the right part [18]. When $\gamma$ is integer there is no aperiodic multimode as $\sin (\gamma \pi)=0$ is a factor of the right part.

Although classical rational orthogonal functions span completely $L_{2}[0, \infty$, they are less appropriate for a good approximation of the polynomially decaying behaviors when using a limited number of terms. Hence, fractional orthogonal bases are expected to better approximate fractional models with fewer parameters.

\footnotetext{
1 'Exactly divisible by the same unit [number] an integral number of times' [6].
} 


\subsection{Stability condition}

Matignon [14, theorem 2.21 p.150] has established the stability condition of any commensurable fractional transfer function (3). However, here is a revisited version of his theorem:

Stability theorem: A commensurable $\gamma$-order transfer function $F(s)=S\left(s^{\gamma}\right)=\frac{T\left(s^{\gamma}\right)}{R\left(s^{\gamma}\right)}$, where $T$ and $R$ are two coprime polynomials, is BIBO stable iff

$$
0<\gamma<2
$$

and for every $s \in \mathbb{C}$ such that $R(s)=0$

$$
|\arg (s)|<\gamma \frac{\pi}{2}
$$

Stability conditions (5) and (6) will be necessary when choosing fractional generating functions for the synthesis of the new basis.

\subsection{Fractional transfer functions belonging to $\mathrm{H}_{2}\left(\mathbb{C}^{+}\right)$}

Contrary to rational systems, the stability condition does not guarantee that a fractional transfer function belongs to $H_{2}\left(\mathbb{C}^{+}\right)$. The $H_{2}$ norm of fractional systems is extensively studied in [12], where it is demonstrated that a stable fractional transfer function as defined in (3), where conditions (5) and (6) are satisfied, belongs to $H_{2}\left(\mathbb{C}^{+}\right)$iff its relative degree is greater than $\frac{1}{2}$ :

$$
\beta_{m_{B}}-\alpha_{m_{A}}>\frac{1}{2}
$$

Condition (7) will be necessary when choosing fractional generating functions for the synthesis of the new basis.

\subsection{Scalar product, orthogonality and Laguerre func- tions}

Laguerre, Kautz and GOB functions form complete orthonormal bases in $L_{2}[0, \infty[$, according to the usual definition of the scalar product:

$$
\left\langle l_{n}, l_{m}\right\rangle=\int_{0}^{\infty} l_{n}(t) l_{m}(t) d t=\delta_{n m}
$$

where the reciprocal in the frequency domain is obtained by Plancherel's theorem:

$$
\left\langle L_{n}, L_{m}\right\rangle=\frac{1}{2 \pi j} \int_{-j \omega}^{j \infty} L_{n}(j \omega) \overline{L_{m}(j \omega)} d \omega=\delta_{n m}
$$

Any function $f(t) \in L_{2}[0, \infty[$, thus satisfying:

$$
\langle f, f\rangle^{\frac{1}{2}}=\|f\|_{2}<\infty
$$

can be written as a linear combination of these functions:

$$
F(s)=\sum_{n=0}^{\infty} a_{n} L_{n}(s)
$$

$F(s)$ is the Laplace transform of $f(t)$. Usually, (11) is truncated to a given order $N$ which is justified by the convergence of Fourier coefficients as $n$ tends to infinity. $F(s)$ is hence approximated by the finite sum:

$$
F(s) \approx F_{N}(s)=\sum_{n=0}^{N} a_{n} L_{n}(s)
$$

The Fourier coefficients are computed by minimizing the least squares criterion:

$$
J=\int_{0}^{\infty}\left(f(t)-f_{N}(t)\right)^{2} d t
$$

which corresponds to the $L_{2}$ norm of the approximation error, according to the definition of the scalar product (8):

$$
J=\left\|f(t)-f_{N}(t)\right\|_{2}^{2}
$$

Minimizing $J$ and taking advantage of the orthonormality, Fourier coefficients are obtained by computing the scalar product in time or frequency domains:

$$
a_{n}=\left\langle f, l_{n}\right\rangle=\left\langle F, L_{n}\right\rangle
$$

As stated previously $l_{n}(t)$ can represent any set of orthonormal functions in $L_{2}[0, \infty[$. In the case where Laguerre functions are used, $l_{n}(t)$ is defined as:

$$
l_{n}(t)=\sqrt{2 \lambda} \frac{e^{\lambda t}}{n !} \frac{d^{n}\left(t^{n} e^{-2 \lambda t}\right)}{d t^{n}}
$$

It has the following Laplace transform:

$$
L_{n}(s)=\sqrt{2 \lambda} \frac{(s-\lambda)^{n}}{(s+\lambda)^{n+1}}
$$

Laguerre functions are well suited for modelling systems with a dominant time constant, because they have a single pole. Abbott [1] has shown that Laguerre functions (16) are divergent as soon as $n$ becomes non-integer:

$$
\int_{0}^{\infty}\left(l_{n}(t)\right)^{2} d t=\infty, \quad \forall n \in \mathbb{R}^{+*} \backslash \mathbb{N}
$$


Therefore, the generalization of Laguerre functions by simply allowing their differentiation order to be real is not possible.

\section{Construction of the orthonormal basis}

This section contains the main results of the paper.

\subsection{Gram-Schmidt orthogonalization procedure}

Given an arbitrary set of functions $\left\{F_{m}\right\}_{m=m_{0}, m_{0}+1, \ldots M}$, where $F_{m} \in H_{2}\left(\mathbb{C}^{+}\right) \forall m$, the orthonormalized functions $\left\{G_{m}\right\}_{m=m_{0}, m_{0}+1, \ldots M}$ are obtained, according to the Gram-Schmidt procedure, as a linear combination of functions $\left\{F_{m}\right\}_{m=m_{0}, m_{0}+1, \ldots M}$ :

$$
\mathbf{G}=\Delta \times \mathbf{F}
$$

where

$$
\begin{aligned}
\mathbf{G} & =\left[\begin{array}{llll}
G_{m_{0}}(s) & G_{m_{0}+1}(s) & \cdots & G_{M}(s)
\end{array}\right]^{T}, \\
\mathbf{F} & =\left[\begin{array}{llll}
F_{m_{0}}(s) & F_{m_{0}+1}(s) & \cdots & F_{M}(s)
\end{array}\right]^{T},
\end{aligned}
$$

and $\Delta$ is a lower-triangular orthogonalization matrix obtained as follows. Since $\mathbf{G}$ is the vector of orthonormal functions:

$$
\begin{gathered}
\left\langle\mathbf{G}, \mathbf{G}^{T}\right\rangle= \\
{\left[\begin{array}{cccc}
\left\langle G_{m_{0}}, G_{m_{0}}\right\rangle & \left\langle G_{m_{0}}, G_{m_{0}+1}\right\rangle & \cdots & \left\langle G_{m_{0}}, G_{M}\right\rangle \\
\left\langle G_{m_{0}+1}, G_{m_{0}}\right\rangle & \ddots & \vdots & \vdots \\
\vdots & \cdots & \ddots & \vdots \\
\left\langle G_{M}, G_{m_{0}}\right\rangle & \left\langle G_{M}, G_{m_{0}+1}\right\rangle & \cdots & \left\langle G_{M}, G_{M}\right\rangle
\end{array}\right]=\mathbf{I}}
\end{gathered}
$$

I denotes $M$ by $M$ identity matrix. Thus, using (19):

$$
\left\langle\mathbf{G}, \mathbf{G}^{T}\right\rangle=\Delta\left\langle\mathbf{F}, \mathbf{F}^{T}\right\rangle \Delta^{T}=\mathbf{I}
$$

The solution of the previous quadratic form is given by Cholesky decomposition $\mathscr{C}$ of the inverse Gram-matrix $\left\langle\mathbf{F}, \mathbf{F}^{T}\right\rangle$ :

$$
\Delta=\mathscr{C}\left(\left\langle\mathbf{F}, \mathbf{F}^{T}\right\rangle^{-1}\right)
$$

As a result, the functions of the orthonormal set are given by:

$$
\mathbf{G}=\mathscr{C}\left(\left\langle\mathbf{F}, \mathbf{F}^{T}\right\rangle^{-1}\right) \times \mathbf{F}
$$

Evaluating the Gram-matrix $\left\langle\mathbf{F}, \mathbf{F}^{T}\right\rangle$ requires, first of all, generating functions to be defined.

\subsection{Generating functions}

To extrapolate Laguerre functions to any fractional order derivative, the $\left\{F_{m}(s)\right\}_{m \geq m_{0}} m \in \mathbb{N}$ set of generating functions is chosen where:

$$
F_{m}(s)=\frac{1}{\left(s^{\gamma}+\lambda\right)^{m}}
$$

and

$$
\left.\lambda \in \mathbb{R}^{+*}, \quad \gamma \in\right] 0,2[
$$

Both conditions in (27) stem from the stability theorem in 2.3, and with impulse response of (26) set to be realvalued. To guarantee that the generating function (26) belongs to $H_{2}\left(\mathbb{C}^{+}\right)$, an additional condition is obtained by applying $(7)$ on $(26)$ :

$$
\gamma m>\frac{1}{2}
$$

Keeping in mind that $m$ is integer, yields:

$$
m \geq m_{0}=\left\lfloor\frac{1}{2 \gamma}\right\rfloor+1
$$

Condition (29) defines the starting value $m_{0}$ of the subscript $m$ to orthogonalize the set of functions $F_{m}(s)$ defined in (26).

\subsection{Evaluating elements of the Gram-matrix $\left\langle\boldsymbol{F}, \boldsymbol{F}^{T}\right\rangle$}

Each element $\left\langle F_{h}, F_{m}\right\rangle$ of the matrix $\left\langle\mathbf{F}, \mathbf{F}^{T}\right\rangle$ is a scalar product of two generating functions $(26)$ :

$$
\begin{aligned}
& \left\langle F_{h}, F_{m}\right\rangle=\frac{1}{2 \pi} \int_{-\infty}^{\infty} F_{h}(j \omega) \overline{F_{m}(j \omega)} d \omega \\
& =\frac{1}{2 \pi} \int_{-\infty}^{\infty}\left(\frac{1}{(j \omega)^{\gamma}+\lambda}\right)^{h}\left(\frac{1}{(-j \omega)^{\gamma}+\lambda}\right)^{m} d \omega
\end{aligned}
$$

Evaluating integral (30) is not an easy task because $F_{m}(s)$ is a complex multivalued function when $\gamma \neq 1$. Consequently, a plane cut is necessary. Integral (30) is solved in appendix A.

\subsection{Completeness of the fractional basis}

Completeness theorem: Define $F_{m}(s)$ as in (26) with conditions (27) satisfied. Then, the linear span of $\left\{F_{m}\right\}_{m=m_{0}, \ldots, \infty}$, where $m_{0}=\left\lfloor\frac{1}{2 \gamma}\right\rfloor+1$, is dense in $H_{2}\left(\mathbb{C}^{+}\right)$. 


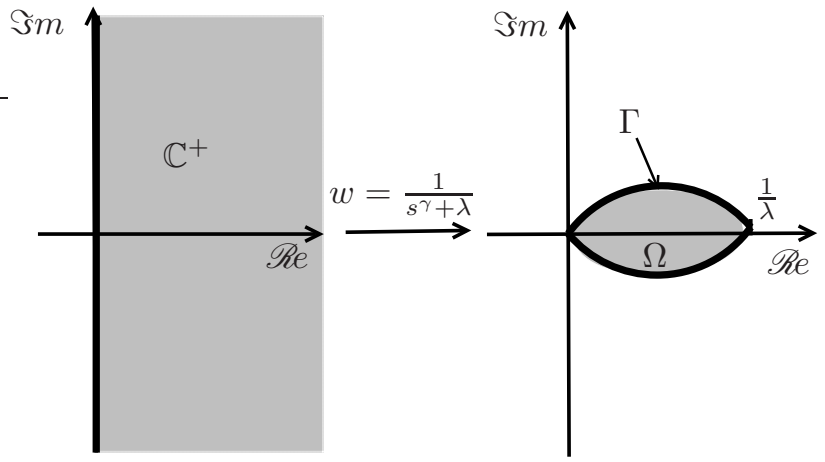

Fig. 1. Conformal mapping $\frac{1}{s^{\gamma}+\lambda}$ of $\mathbb{C}^{+}$onto $\Omega$ for $0<\gamma<1$. When $1<\gamma<2, \Omega$ intrudes in $\mathbb{C} \backslash \overline{\mathbb{C}^{+}}$.

Proof: To prove the completeness of the generating functions $\left\{F_{m}\right\}_{m=m_{0}, \ldots, \infty}$ in $H_{2}\left(\mathbb{C}^{+}\right)$, it suffices to prove the completeness in a dense subset of $\mathrm{H}_{2}\left(\mathbb{C}^{+}\right)$, denoted $\mathcal{H}$, since for a given $\varepsilon>0$ and $K \in H_{2}\left(\mathbb{C}^{+}\right)$, there exists an $H \in \mathcal{H}$ satisfying:

$$
\|K-H\|_{2}<\varepsilon
$$

For a given positive integer $N$, there exists a dense subset $\mathcal{H}$ of $H_{2}\left(\mathbb{C}^{+}\right)$satisfying [9, Corollary 3.3$]$ :

(i) $H(s)$ is continuous on $\overline{\mathbb{C}^{+}}$and infinitely differentiable on the imaginary axis for all $H \in \mathcal{H}$.

(ii) $\lim _{|s| \rightarrow \infty}|s|^{N}|H(s)|=0, \forall s \in \overline{\mathbb{C}^{+}}$.

For a given $H \in \mathcal{H}$, let

$$
G(s)=\left(s^{\gamma}+\lambda\right)^{m_{0}} H(s)
$$

Due to (i) and by setting $N>\gamma m_{0}$ in (ii), $G$ is analytic on $\mathbb{C}^{+}$, continuous on $\overline{\mathbb{C}^{+}}$and vanishes when $|s| \rightarrow \infty$.

Let $w=F_{1}(s)=\frac{1}{s^{\gamma}+\lambda} \cdot F_{1}$ is a bijective conformal mapping from $\mathbb{C}^{+}$on bounded open domain $\Omega$ limited by Jordan curve $\Gamma$, where $\Gamma$ is a union of two circular arcs with end points 0 and $\frac{1}{\lambda}$, as shown in fig. 1 .

Define

$$
\mathcal{F}(z)=G \circ F_{1}^{-1}(z), \quad z \in \Omega
$$

Thus, $\mathcal{F}$ is analytic on $\Omega$ and continuous on its closure $\bar{\Omega}$. All the assumptions in Mergelyan's theorem [20, theorem 20.5] are satisfied and there is a polynomial $P(z)=\sum_{n=0}^{N} a_{n} z^{n}$ such that:

$$
|\mathcal{F}(z)-P(z)| \leq \epsilon, \quad \forall z \in \bar{\Omega}
$$

which, replacing $z$ by $F_{1}(s)$, yields

$$
\left|G(s)-\sum_{n=0}^{N} a_{n}\left(F_{1}(s)\right)^{n}\right| \leq \epsilon, \quad \forall s \in \overline{\mathbb{C}^{+}}
$$

Replacing $G(s)$ by its definition (31), noting that $F_{n}(s)=\left(F_{1}(s)\right)^{n}$, and integrating the square of the obtained expression along the imaginary axis, yields the desired result:

$$
\begin{gathered}
\int_{-j \infty}^{j \infty}\left|H(s)-\sum_{n=0}^{N} a_{n} F_{n+m_{0}}(s)\right|^{2} d s \leq \epsilon^{2} \int_{-j \infty}^{j \infty}\left|F_{m_{0}}(s)\right|^{2} d s \\
\left\|H(s)-\sum_{n=0}^{N} a_{n} F_{n+m_{0}}(s)\right\|_{2} \leq \varepsilon
\end{gathered}
$$

Thus, the linear span of $\left\{F_{m}\right\}_{m_{0}, m_{0}+1, \ldots, \infty}$ is dense in $H_{2}\left(\mathbb{C}^{+}\right)$, which completes the proof.

Consequently, the linear span $\left\{G_{m}\right\}_{m \geq m_{0}}$ is dense in $H_{2}\left(\mathbb{C}^{+}\right)$too, and the orthonormal functions $\left\{G_{m}\right\}_{m \geq m_{0}}$ can be used to approximate any finite energy system.

\section{Important remarks}

- The new basis has two tuning parameters: an eigenvalue $\lambda$ and a commensurable order $\gamma$.

- The only necessary conditions to build the basis are $\lambda>0$ and $0<\gamma<2$.

- When the commensurable order $\gamma$ equals 1 , the built basis corresponds exactly to the classical Laguerre basis. Hence, the fractional Laguerre basis can be considered as an extrapolation of the rational Laguerre basis for any commensurable order $\gamma \in] 0,2[$.

- For a large number of functions (large $M$ ), bad conditioning of $\left\langle\mathbf{F}, \mathbf{F}^{T}\right\rangle$ matrix (to be inverted in (24)) may occur. Orthogonal functions can then be computed using a recursive Gram-Schmidt procedure:

$$
\left\{\begin{array}{rl}
G_{m_{0}}(s) & =\frac{F_{m_{0}}(s)}{\left\|F_{m_{0}}(s)\right\|} \\
G_{m}(s) & =\frac{F_{m}(s)-\sum_{i=m_{0}}^{m-1}\left\langle F_{m}(s), G_{i}(s)\right\rangle G_{i}(s)}{\left\|F_{m}(s)-\sum_{i=m_{0}}^{m-1}\left\langle F_{m}(s), G_{i}(s)\right\rangle G_{i}(s)\right\|}, \\
\text { for } m>m_{0} & m,
\end{array}\right.
$$




\subsection{Examples}

When the differentiation order $\gamma=0.4$ and the eigenvalue $\lambda=1.5$, condition (29) imposes starting from $m_{0}=2$. Hence, the first three orthonormal functions are:

$$
\begin{aligned}
G_{2}(s) & =\frac{3.18}{\left(s^{0.4}+1.5\right)^{2}} \\
G_{3}(s) & =\frac{4.62}{\left(s^{0.4}+1.5\right)^{2}}+\frac{-18.48}{\left(s^{0.4}+1.5\right)^{3}} \\
G_{4}(s) & =\frac{5.87}{\left(s^{0.4}+1.5\right)^{2}}+\frac{-53.05}{\left(s^{0.4}+1.5\right)^{3}}+\frac{95.50}{\left(s^{0.4}+1.5\right)^{4}}
\end{aligned}
$$

When the differentiation order is integer, $\gamma=1$, and the eigenvalue $\lambda=1.5$, the first three orthonormal functions are:

$$
\begin{aligned}
& G_{1}(s)=\frac{1.73}{s+1.5} \\
& G_{2}(s)=\frac{1.73}{s+1.5}+\frac{-5.19}{(s+1.5)^{2}} \\
& G_{3}(s)=\frac{1.73}{s+1.5}+\frac{-10.39}{(s+1.5)^{2}}+\frac{15.59}{(s+1.5)^{3}}
\end{aligned}
$$

This corresponds exactly to the classical definition of Laguerre functions (17), which are thus considered as a special case of the new fractional basis when $\gamma=1$.

When the differentiation order $\gamma=1.5$ and the eigenvalue $\lambda=1.5$, the first three orthonormal functions are:

$$
\begin{aligned}
G_{1}(s) & =\frac{1.49}{s^{1.5}+1.5} \\
G_{2}(s) & =\frac{1.06}{s^{1.5}+1.5}+\frac{-2.38}{\left(s^{1.5}+1.5\right)^{2}} \\
G_{3}(s) & =\frac{1.06}{s^{1.5}+1.5}+\frac{-2.77}{\left(s^{1.5}+1.5\right)^{2}}+\frac{3.56}{\left(s^{1.5}+1.5\right)^{3}}
\end{aligned}
$$

Fig. 2 shows impulse responses of the first three orthogonal functions for $\lambda=1.5$ and various values of $\gamma$. One can notice that fractional orthonormal functions are more oscillatory when the differentiation order increases beyond 1 . Also the impulse response at $t=0$ can be finite or infinite depending on $\gamma$. Proof is given by the initial value theorem:

$$
\lim _{t \rightarrow 0} g_{m}(t)=\lim _{s \rightarrow \infty} s G_{m}(s)= \begin{cases}\infty & \text { if } 0<\gamma<1 \\ \sqrt{2 \lambda} & \text { if } \gamma=1 \\ 0 & \text { if } 1<\gamma<2\end{cases}
$$

\section{System identification using fractional La- guerre basis}

Fractional Laguerre basis can be used in output error identification with fixed denominator models [22]. Prior knowledge can be used to fix both tuning parameters $\gamma$ and $\lambda$. These two parameters are then plugged in (26), and the orthonormal basis is synthesized using (25).
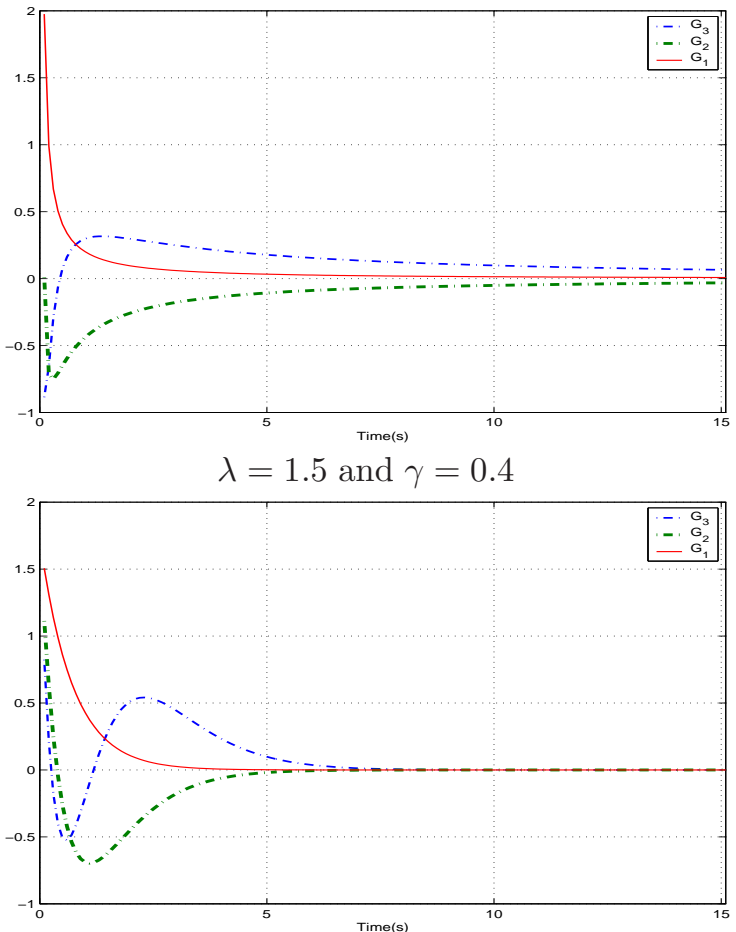

$\lambda=1.5$ and $\gamma=1$

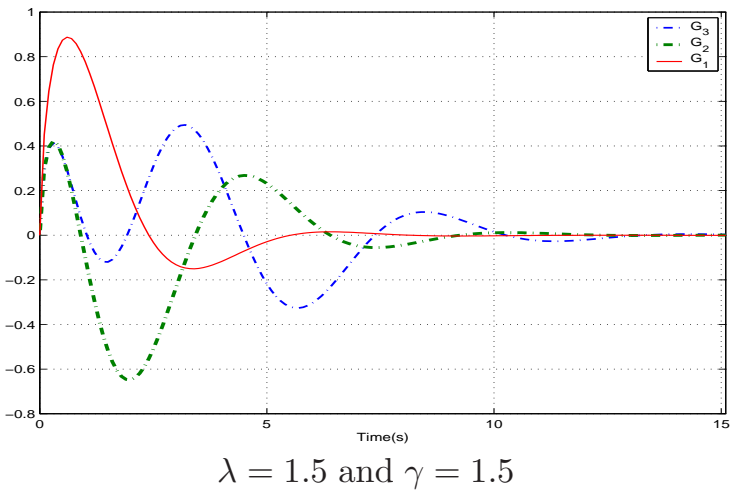

Fig. 2. Impulse responses of the first three orthogonal functions for $\lambda=1.5$ and for different values of $\gamma$

Then, Fourier coefficients are computed using a least squares estimate.

Assume $u(t), y(t), t \in[0, T]$ input and output data generated using a finite energy linear fractional model $H$. So $H(s)$ can be approximated by a linear combination of orthonormal functions with:

$$
H(s) \approx \sum_{m=m_{0}}^{M} g_{m} G_{m}(s)=\mathbf{g}^{T} \mathbf{G}(s)
$$

where $\mathbf{G}$ is defined by (20) and

$$
\mathbf{g}=\left[\begin{array}{llll}
g_{m_{0}} & g_{m_{0}+1} & \ldots & g_{M}
\end{array}\right]^{T}
$$

The truncation order $M$ is fixed to obtain a satis- 
factory approximation. Akaike and Young information criteria can be used. The identification procedure consists of computing optimal coefficient vector $g=\left[g_{m_{0}}, g_{m_{0}+1}, \ldots, g_{M}\right]^{T}$ which minimizes the least square error:

$$
J=\frac{1}{T} \int_{0}^{T}(\varepsilon(t))^{2} d t=\langle\varepsilon, \varepsilon\rangle
$$

where

$$
\varepsilon(t)=y(t)-\sum_{m=m_{0}}^{M} g_{m} u_{G_{m}}(t)
$$

$y(t)$ and $u_{G_{m}(t)}$ are respectively the system and orthogonal network outputs:

$$
u_{G_{m}}(t)=G_{m}(t) \otimes u(t)
$$

Setting:

$$
\mathbf{u}_{\mathbf{G}}(t)=\left[u_{G_{m_{0}}}(t) u_{G_{m_{0}+1}}(t) \cdots u_{G_{M}}(t)\right],
$$

the optimum estimation of Fourier coefficients $\hat{\mathrm{g}}$ is given by the least squares formula:

$$
\hat{\mathbf{g}}=\left[\int_{0}^{T} \mathbf{u}_{\mathbf{G}}(t)^{T} \mathbf{u}_{\mathbf{G}}(t) d t\right]^{-1} \int_{0}^{T} \mathbf{u}_{\mathbf{G}}(t)^{T} y(t) d t
$$

Or, after a numerical discretization, by defining $\mathbf{Y}$ as a column vector of system's output and $\boldsymbol{\Phi}$ as a regression matrix where columns are filter outputs, $\hat{\mathrm{g}}$ can be approximated by:

$$
\hat{\mathrm{g}}=\left(\boldsymbol{\Phi}^{T} \boldsymbol{\Phi}\right)^{-1} \boldsymbol{\Phi}^{T} \mathbf{Y}
$$

All properties of least squares estimates (persistent excitation, variance on estimates) as stated in [11] apply in this context.

\section{Example}

To illustrate the use of fractional Laguerre bases in system identification, the following academic system is simulated and then identified in a noisy context:

$$
H(s)=\frac{1}{s^{0.7}+2}+\frac{1}{s^{0.8}+2}+\frac{1}{s^{0.9}+1}
$$

The pseudo random binary sequence used as input signal and the output signal are plotted in fig. 3. Output signal is corrupted by a stationary zero mean Gaussian white noise with a signal to noise ratio arbitrarily set to $10 \log _{10}\left(\frac{\text { Signal energy }}{\text { Noise energy }}\right)=13 \mathrm{~dB}$.
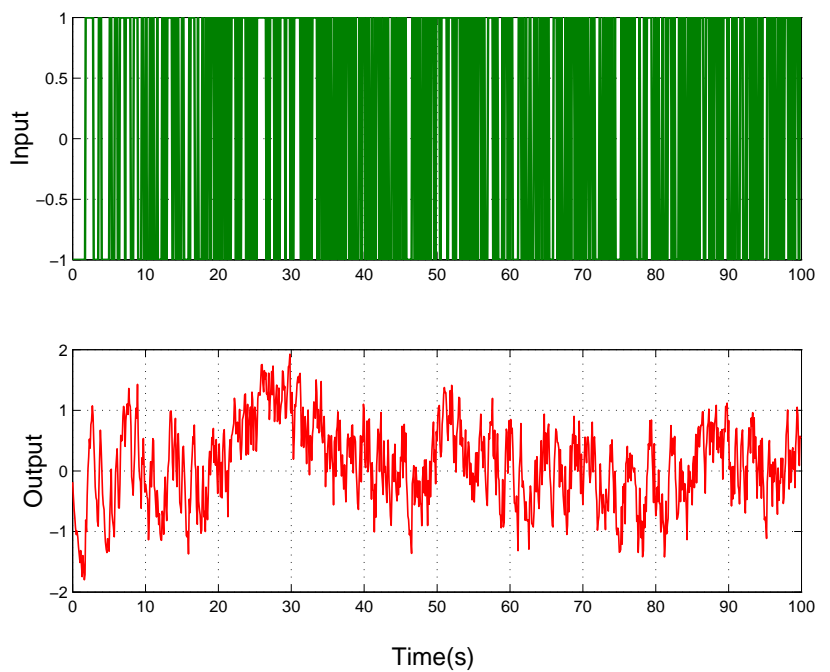

Fig. 3. Input and output identification data

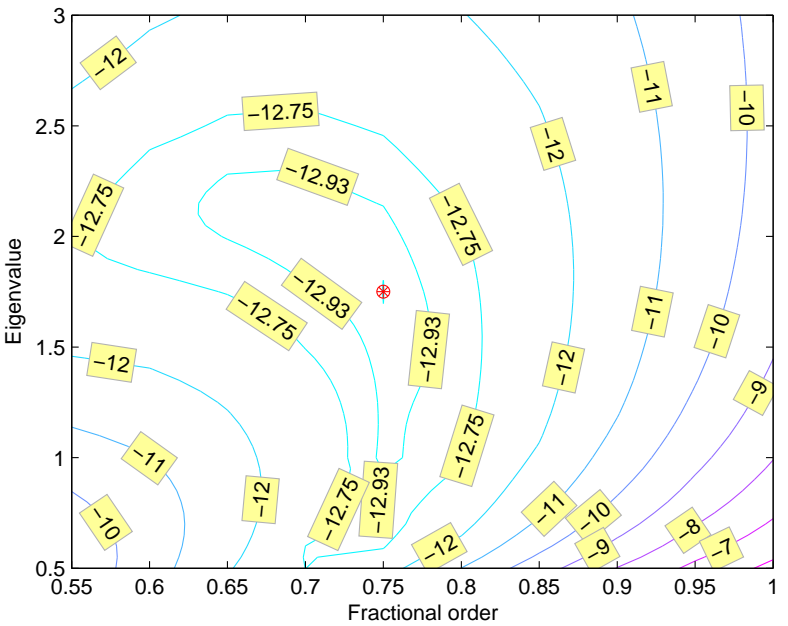

Fig. 4. Isocontour of the least square error in $\mathrm{dB}$

$H$ is approximated using two fractional Laguerre functions. Least squares error, $J$, is computed in terms of differentiation order $\gamma$ varying from 0.55 to 1.9 and eigenvalue $\lambda$ varying from 0.55 to 3 . For each pair of $(\gamma, \lambda)$, generating functions (26) are orthogonalized, and then Fourier coefficients, $\mathbf{g}$, computed using the least squares formula (42). Iso-contours of $J$ are plotted in fig. 4 for $\gamma \in$ $[0.55,1]$ and $\lambda \in[0.5,3]$. Then, tuning parameters are chosen around the optimal values $(\gamma, \lambda)=(0.75,1.75)$. The optimal differentiation order is far from an integer value, as expected, because the initial system (44) is fractional. The optimal model is:

$$
\hat{H}(s)=1.90 G_{1}(s)-0.29 G_{2}(s)
$$




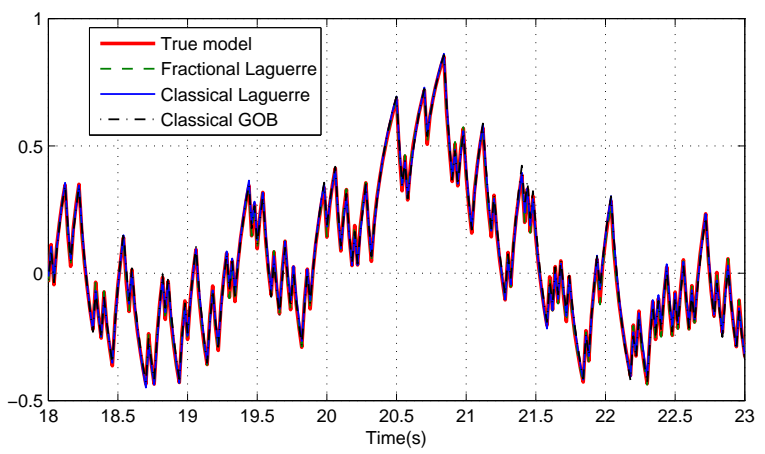

Fig. 5. Validation output data - The difference between the true system, the fractional Laguerre model, the Laguerre model and the generalized orthogonal basis model is almost indistinguishable.

where $G_{1}(s)$ and $G_{2}(s)$ are the orthonormal functions:

$$
\begin{aligned}
G_{1}(s) & =\frac{1.51}{s^{0.75}+1.75} \\
G_{2}(s) & =\frac{1.34 s^{0.75}-4.68}{s^{1.50}+3.50 s^{0.75}+3.06}
\end{aligned}
$$

The normalized residual,

$$
J_{\mathrm{dB}}=10 \log \left(\frac{\int_{0}^{T}(\varepsilon(t))^{2} d t}{\int_{0}^{T}(y(t))^{2} d t}\right) \approx-13 \mathrm{~dB}
$$

is mainly due to the injected noise (as SNR is $13 \mathrm{~dB}$ ). The modeling error is hence very small, which is confirmed when noise-free validation data are applied to the true system and to the obtained model.

For further comparison, the system is identified using classical orthogonal bases. Six Laguerre functions or two GOB functions with optimal poles provide similar approximation errors: $J_{\mathrm{dB}}=-12.5 \mathrm{~dB}$. Optimal Kautz poles converge to the optimal Laguerre pole (the imaginary parts equal zero). Fig. 5 shows that time-domain outputs of the true system (44) and the identified models are almost identical. A better distinction is obtained in the frequency domain and fig. 6 shows that the fractional Laguerre model clearly exhibits a better approximation. Fractional models can indeed have any asymptotic slope in the gain diagram and any asymptotic phase lock in the phase diagram [18].

\section{CONCLUSION}

An orthonormal fractional Laguerre basis has been synthesized for system approximations. It has two tuning
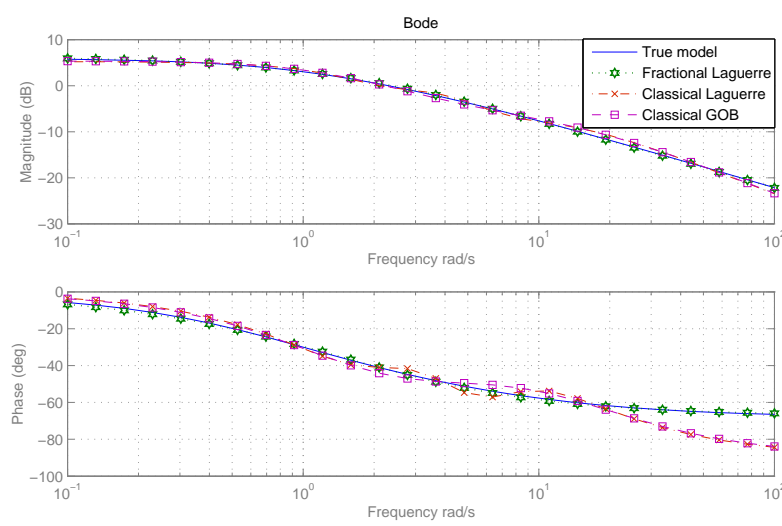

Fig. 6. Bode diagrams of the true system, the fractional Laguerre model, the Laguerre model, and the GOB model.

parameters: an eigenvalue and a commensurable differentiation order $\gamma \in] 0,2[$. Classical Laguerre functions are shown as a special case of the new basis corresponding to $\gamma=1$. This is the first fractional basis ever developed. An academic system identification example shows the advantage of using a fractional basis as compared to a rational one.

\section{A Computing scalar product $\left\langle F_{h}, F_{m}\right\rangle$}

$F_{h}$ and $F_{m}$ are the orthogonalized functions defined by (26).

$$
\left\langle F_{h}, F_{m}\right\rangle=\left\langle\frac{1}{\left(s^{\gamma}+\lambda\right)^{h}}, \frac{1}{\left(s^{\gamma}+\lambda\right)^{m}}\right\rangle
$$

where $\lambda \in \mathbb{R}^{+*}, \gamma \in \mathbb{R}^{+*}, h \geq m_{0}, m \geq m_{0}$ and $m_{0}=$ $\left\lfloor\frac{1}{2 \gamma}\right\rfloor+1$.

$$
\left\langle F_{h}, F_{m}\right\rangle=\frac{1}{2 \pi} \int_{-\infty}^{+\infty} \frac{1}{\left((j \omega)^{\gamma}+\lambda\right)^{h}}\left(\frac{1}{\left((j \omega)^{\gamma}+\lambda\right)^{m}}\right) d \omega
$$

then

$$
\begin{aligned}
\left\langle F_{h}, F_{m}\right\rangle= & \frac{1}{2 \pi} \int_{0}^{\infty} \frac{d \omega}{\left((j \omega)^{\gamma}+\lambda\right)^{h}\left(\bar{j}^{\gamma} \omega^{\gamma}+\lambda\right)^{m}} \\
& +\frac{1}{2 \pi} \int_{0}^{\infty} \frac{d \omega}{\left((-j \omega)^{\gamma}+\lambda\right)^{h}\left(\overline{(-j)^{\gamma}} \omega^{\gamma}+\lambda\right)^{m}}
\end{aligned}
$$

The following change of variable is applied: $\omega^{\gamma}=x$, $d \omega=\frac{1}{\gamma} x^{\frac{1}{\gamma}-1} d x$. Also, by defining:

$$
I\left(\zeta, \eta, \frac{1}{\gamma}, h, m\right)=\int_{0}^{\infty} \frac{x^{\frac{1}{\gamma}-1} d x}{(\zeta x+1)^{h}(\eta x+1)^{m}}
$$


(A.3) can be rewritten as:

$$
\begin{aligned}
\left\langle F_{h}, F_{m}\right\rangle= & \frac{1}{\gamma 2 \pi \lambda^{h+m}}\left[I\left(\lambda^{-1} e^{j \frac{\pi}{2} \gamma}, \lambda^{-1} e^{-j \frac{\pi}{2} \gamma}, \frac{1}{\gamma}, h, m\right)\right. \\
& \left.+I\left(\lambda^{-1} e^{-j \frac{\pi}{2} \gamma}, \lambda^{-1} e^{j \frac{\pi}{2} \gamma}, \frac{1}{\gamma}, h, m\right)\right]
\end{aligned}
$$

Let $q=\left\lfloor\frac{1}{\gamma}\right\rfloor$ and $\rho$ be the non-integer part of $\frac{1}{\gamma}$.

Depending on the value of $\gamma$, integral $I$ is computed differently. Hence the following two decompositions:

- If $0<\gamma<2$ and $\frac{1}{\gamma} \notin \mathbb{N}$

$$
I\left(\zeta, \eta, \frac{1}{\gamma}, h, m\right)=\int_{0}^{\infty}\left(\sum_{l=0}^{h-1} \frac{A_{l} x^{\rho-1}}{(\zeta x+1)^{h-l}}+\sum_{l=0}^{m-1} \frac{B_{l} x^{\rho-1}}{(\eta x+1)^{m-l}}\right) d x
$$

where

$$
\begin{aligned}
& A_{l}=\frac{q !}{(m-1) ! \zeta^{l}} \times \\
& \sum_{k=\operatorname{Max}(0, l-q)}^{l}\left(\frac{(m+k-1) !}{k !(l-k) !(q+k-l) !} \frac{(-\eta)^{k}(-\zeta)^{l-q-k}}{\left(-\frac{\eta}{\zeta}+1\right)^{m+k}}\right)
\end{aligned}
$$

and

$$
\begin{aligned}
& B_{l}=\frac{q !}{(h-1) ! \eta^{l}} \times \\
& \sum_{k=\operatorname{Max}(0, l-q)}^{l}\left(\frac{(h+k-1) !}{k !(l-k) !(q+k-l) !} \frac{(-\zeta)^{k}(-\eta)^{l-q-k}}{\left(-\frac{\zeta}{\eta}+1\right)^{h+k}}\right)
\end{aligned}
$$

By applying formula [3.194, 4 p. 285] of [10],

$$
\int_{0}^{\infty} \frac{x^{\rho-1} d x}{(\zeta x+1)^{l}}=\frac{\pi(-1)^{l-1}}{\zeta^{\rho} \sin (\rho \pi)}\left(\begin{array}{c}
\rho-1 \\
l-1
\end{array}\right), \text { if } 1>\rho
$$

The integral (A.6) is:

$$
\begin{aligned}
& I\left(\zeta, \eta, \frac{1}{\gamma}, h, m\right)= \\
& \frac{\pi}{\zeta^{\rho} \sin (\rho \pi)} \sum_{l=0}^{h-1} A_{l}(-1)^{h-l-1}\left(\begin{array}{l}
\rho-1 \\
h-l-1
\end{array}\right)+ \\
& \frac{\pi}{\eta^{\rho} \sin (\rho \pi)} \sum_{l=0}^{m-1} B_{l}(-1)^{m-l-1}\left(\begin{array}{l}
\rho-1 \\
m-l-1
\end{array}\right)
\end{aligned}
$$

- If $0<\gamma<2$ and $\frac{1}{\gamma} \in \mathbb{N}$

$$
\begin{aligned}
& I\left(\zeta, \eta, \frac{1}{\gamma}, h, m\right)=\int_{0}^{\infty}\left[\frac{A_{h-1}^{\prime}+B_{m-1}^{\prime}}{(\zeta x+1)(\eta x+1)}\right. \\
& \left.+\sum_{l=0}^{h-2} \frac{A_{l}^{\prime}}{(\zeta x+1)^{h-l}}+\sum_{l=0}^{m-2} \frac{B_{l}^{\prime}}{(\eta x+1)^{m-l}}\right] d x
\end{aligned}
$$

where

$$
\begin{aligned}
& A_{l}^{\prime}=\frac{(q-1) !}{(m-1) ! \zeta^{l}} \times \sum_{k=\operatorname{Max}(0, l-q+1)}^{l} \\
& \left(\frac{(m+k-1) !}{k !(l-k) !(q+k-l-1) !} \frac{(-\eta)^{k}(-\zeta)^{l-q-k+1}}{\left(-\frac{\eta}{\zeta}+1\right)^{m+k}}\right)
\end{aligned}
$$

and

$$
\begin{aligned}
& B_{l}^{\prime}=\frac{(q-1) !}{(h-1) ! \eta^{l}} \sum_{k=\operatorname{Max}(0, l-q+1)}^{l} \\
& \left(\frac{(h+k-1) !}{k !(l-k) !(q+k-l-1) !} \frac{(-\zeta)^{k}(-\eta)^{l-q-k+1}}{\left(-\frac{\zeta}{\eta}+1\right)^{h+k}}\right)
\end{aligned}
$$

Integral $I$ can be computed using:

$$
\int_{0}^{\infty} \frac{d x}{(\zeta x+1)^{l}}=\frac{1}{\zeta(l-1)}, \quad \text { if } 1>1
$$

and

$$
\int_{0}^{\infty} \frac{d x}{(\zeta x+1)(\eta x+1)}=\frac{\ln (\zeta)-\ln (\eta)}{\zeta-\eta}
$$

Integral (A.11) is now:

$$
\begin{aligned}
& I\left(\zeta, \eta, \frac{1}{\gamma}, h, m\right)=\frac{\left(A_{h-1}^{\prime}+B_{m-1}^{\prime}\right)}{\zeta-\eta}(\ln (\zeta)-\ln (\eta)) \\
& \quad+\sum_{l=0}^{h-2} \frac{A_{l}^{\prime}}{\zeta(h-l-1)}+\sum_{l=0}^{m-2} \frac{B_{l}^{\prime}}{\eta(m-l-1)} \quad(\mathrm{A} .16)
\end{aligned}
$$

The scalar product (A.1) can be deduced from (A.5) and (A.10) when $\frac{1}{\gamma} \notin \mathbb{N}$, and from (A.5) and (A.16) when $\frac{1}{\gamma} \in \mathbb{N}$. 


\section{References}

[1] P.C. Abbott. Generalized Laguerre polynomials and quantum mechanics. Journal of Physics A: Mathematical and General, 33(42):7659-7660, 2000.

[2] H. Akçay and B. Ninness. Orthonormal basis functions for modelling continuous-time systems. Signal processing, $77: 261-274,1999$.

[3] M. Aoun. Systèmes linéaires non entiers et identification par bases orthogonales non entières. $\mathrm{PhD}$ thesis, Université Bordeaux 1, France, 2005.

[4] M. Aoun, R. Malti, F. Levron, and A. Oustaloup. Numerical simulations of fractional systems: An overview of existing methods and improvements. International Journal of Nonlinear Dynamics and Chaos in Engineering Systems, Special Issue on 'Fractional Derivatives and Their Applications', 38(1-4):117-131, 2004.

[5] J.-L. Battaglia, L. Le Lay, J.-C. Batsale, A. Oustaloup, and O. Cois. Heat flux estimation through inverted non integer identification models. International Journal of Thermal Sciences, 39(3):374-389, 2000.

[6] The American Heritage (C), editor. Dictionary of the English Language. Houghton Mifflin Company, forth edition, 2000.

[7] R. Darling and J. Newman. On the short behavior of porous intercalation electrodes. J. Electrochem. Soc., 144(9):30573063, 1997 .

[8] A.-M. El Sayed. On the generalized Laguerre polynomials of arbitrary (fractional) orders and quantum mechanics. Journal of Physics A: Mathematical and General, 32:86478654, 1999

[9] J. B. Garnett. Bounded analytic functions. Springer, revised first edition, 2007.

[10] I.-S. Gradshteyn and I.-M. Ryshik. Table of integrals, series, and products. Academic Press, 1980.

[11] L. Ljung. System identification - Theory for the user. Prentice Hall, $2^{\text {nd }}$ edition, 1999.

[12] R. Malti, M. Aoun, F. Levron, and A. Oustaloup. $H_{2}$ norm of fractional differential systems. In Proceedings of Design Engineering Technical Conferences and Computer and Information in Engineering Conferences, Chicago, Illinois, USA, sep 2003. ASME.

[13] R. Malti, S.-B. Ekongolo, and J. Ragot. Dynamic SISO and MIMO system approximations based on optimal Laguerre models. IEEE Transactions on Automatic Control, 43(9), 1998.

[14] D. Matignon. Stability properties for generalized fractional differential systems. ESAIM proceedings Systèmes Différentiels Fractionnaires - Modèles, Méthodes et Applications, 5:145-158, 1998.

[15] B. Ninness and F. Gustafsson. A unifying construction of orthonormal bases for system identification. IEEE Transactions on Automatic Control, 42(4):515-521, 1997.

[16] K.-B. Oldham and J. Spanier. The Fractional Calculus: Theory and Applications of Differentiation and Integration to Arbitrary Order. Academic Press, New-York et London, 1974 .

[17] M.D. Ortigueira and J.A. Tenreiro Machado, editors. Signal Processing - special issue: Fractional calculus applications in signals and systems, volume 86. Elsevier, October 2006.

[18] A. Oustaloup. La dérivation non entière: théorie, synthèse et applications. Hermès, Paris, 1995.
[19] T. Poinot and J.-C. Trigeassou. A method for modelling and simulation of fractional systems. Signal processing, 83:23192333, 2003.

[20] W. Rudin. Real and complex analysis. McGraw-Hill, third edition, 1987.

[21] P.-M.-J. Van den Hof, P.-S.-C. Heuberger, and J. Bokor. System identification with generalized orthonormal basis functions. Automatica, 31(12):1821-1834, 1995.

[22] B. Wahlberg. System identification using Laguerre models. IEEE Transactions on Automatic Control, 36:551-562, 1991. 